ansässige Ärzte gegenüber den in Deutschland lebenden Adressaten der Werbung mittels einer App Diagnosen, Therapieempfehlungen und Krankschreibungen durchführen, wobei ,alternativ zum traditionellen Arztbesuch“ der ,,digitale Arztbesuch per App“ angepriesen wird („Bleib einfach im Bett, wenn Du zum Arzt gehst"). Es wird damit geworben, dass „erstmals in Deutschland“ die komplette ärztliche Versorgung, nämlich „Diagnosen, Therapieempfehlung und Krankschreibung", mittels einer App online erfolgen könne (,Alles per App“). Hierzu führen die Bekl. in ihrer Berufungsbegründung aus, dass in rund 90\% der Fälle, weswegen Patienten einen Arzt aufsuchten, eine persönliche Konsultation des Arztes nicht notwendig sei. Diese nicht belegte Behauptung erscheint allerdings wenig nachvollziehbar, nachdem grundsätzlich jeder Krankheitsverdacht nach allgemeinen fachlichen Standards eine Basisuntersuchung erfordert, in der Regel unmittelbar durch Funktionsprüfungen (etwa von Atmung, Kreislauf, Blutdruck) und Besichtigungen, Abtasten, Abklopfen und Abhören des Körpers sowie ggf. der Erhebung weiterer Laborwerte (vgl. Katzenmeier, NJW 2019, 1769 m.w. N - Haftungsrechtliche Grenzen ärztlicher Fernbehandlung). Bei einer ausschließlichen Videokonsultation, wie sie im Streitfall beworben wurde, muss sich der Arzt demgegenüber von vornherein auf eine verkürzte Wahrnehmung bei der Anamnese verlassen. Der im Fall einer ausschließlichen Fernbehandlung naheliegende Vorwurf einer Vernachlässigung der Befunderhebungspflicht muss den behandelnden Arzt grundsätzlich zu Vorsicht und Zurückhaltung veranlassen, so dass auch nach der Reform des $\$ 7$ Abs. 4 MBObzw. der Berufsordnungen der Landesärztekammern (die insoweit unterschiedliche Neuregelungen aufweisen) bei dem geringsten Zweifel umgehend eine persönliche Untersuchung des Patienten zu veranlassen sein wird (vgl. Katzenmeier, a.a.O.). Die im Streitfall beworbene Ersetzung des persönlichen Arztbesuchs durch eine alternative digitale Fernbehandlung per digitaler App ,,von der Diagnose über die Therapieempfehlung bis hin zur Krankschreibung" für nicht näher konkretisierte Behandlungsfälle und -situationen durch in der Schweiz sitzende Ärzte wird von dem Ausnahmetatbestand des $\$ 9$ S. 2 HWG n. F., wonach vorausgesetzt wird, dass ein ärztlicher Kontakt mit dem zu behandelnden Menschen nach allgemein anerkannten Standards in den beworbenen Fällen nicht erforderlich ist, in dieser generellen Weite somit nicht gedeckt. Zwar wird dem von der Werbung angesprochenen potenziellen Patienten, wie von der Bekl. geltend gemacht, grundsätzlich bewusst sein, dass im Wege einer Fernbehandlung in tatsächlicher Hinsicht nur begrenzte Diagnose- und Behandlungsmöglichkeiten des Arztes bestehen, also je nach Krankheitsbild weitergehende Untersuchungen und ärztliche Eingriffe erforderlich sein können. Die streitgegenständliche Werbung berücksichtigt aber nicht, dass auch im Rahmen dieser tatsächlich eingeschränkten Möglichkeiten eine Werbung für Fernbehandlungen nicht generell zulässig ist, sondern nur unter der Voraussetzung, dass bei Einhaltung allgemein anerkannter fachlicher Standards kein persönlicher Kontakt mit dem zu behandelnden Menschen erforderlich ist i.S.d. \$9 S. 2 HWG. Wollte man die hier streitgegenständliche Werbung unter die Ausnahmeregelung des $₫ 9$ S. 2 HWG subsumieren, würde das grundsätzliche Werbeverbot für Fernbehandlungen nach $\$ 9$ S. 1 HWG im Übrigen praktisch leerlaufen.

Soweit sich die angegriffene Werbung der Bekl. auf Krankschreibungen im Wege der Online-Videokonsultation bezieht, ist dem Kl. weiterhin darin zuzustimmen, dass nach allgemeinen fachlichen Standards (s. a. $\$ 25$ S. 1 MBOÄ) ein persönlicher ärztlicher Kontakt mit dem zu behandelnden Menschen - gerade bei Patienten, die dem Arzt persönlich unbekannt sind, wie im streitgegenständlichen Fall - grundsätzlich erforderlich ist i.S.v. \$9 S. $2 \mathrm{HWG}$, so dass die Werbung für generelle Krankschreibungen per Videokonsultation durch im Ausland ansässige Ärzte nicht auf \$9 S. 2 HWG gestützt werden kann (vgl. auch die Wertung des $₫ 4$ Abs. 1 S. 1, S. 2 der Arbeitsunfähigkeits-Richtlinie betreffend gesetzlich Versicherte, Anlage KR 7, S. 5, sowie die Entschließung des 121. Ärztetags 2018, Anlage KR 5, S. 298, wonach die Ausstellung einer Arbeitsunfähigkeitsbescheinigung grundsätzlich abgelehnt wird, wenn es im Rahmen einer Fernbehandlung zu keinem persönlichen Arzt-Patienten-Kontakt kommt).

g) Die Regelung des $\$ 9$ HWG ist dazu bestimmt, im Interesse der Marktteilnehmer das Marktverhalten zu regeln i.S.v. \$3a UWG (vgl. OLG München, GRUR-RR 2012, 435, 436 - Unsere Experten sind für Sie da), wobei eine Verletzung der Bestimmung - welche dem Schutz der Gesundheit der Verbraucher dient - geeignet ist, die Interessen der Verbraucher spürbar i.S.v. \$3 Abs. 1 UWG zu beeinträchtigen (vgl. BGH, GRUR 2012, 647 Rdnr. 42 INJECTIO; OLG München, a.a. O.)

h) Die gemäß $₫ 8$ Abs. 1 S. 1 UWG erforderliche Wiederholungsgefahr wird durch die Verletzungshandlung indiziert und besteht mangels Abgabe einer strafbewehrten Unterlassungserklärung seitens der Bekl. weiterhin fort. Dabei liegt das Charakteristische der angegriffenen Verletzungshandlung unter anderem darin, dass sich die streitgegenständliche Werbung auf Fernbehandlungen bezieht, die an in Deutschland lebenden Patienten durch im Ausland ansässige Ärzte durchgeführt werden, und ist somit die Wiederholungsgefahr - die grundsätzlich auch für kerngleiche Handlungen begründet wird - nicht auf die Bewerbung von Fernbehandlungen durch Ärzte aus der Schweiz beschränkt.

2. $[\ldots]$

https://doi.org/10.1007/s00350-021-5795-0

\section{Anmerkung zu OLG München, Urt. v. 9.7.2020 - 6 U 5180/19 (LG München)}

\section{Julian Braun}

Die vorliegende Entscheidung des OLG München gibt insbesondere in den nachfolgend aufgeführten Punkten Anlass zur Kommentierung:

\section{Zum Verstoß gegen $₫ 9$ HWG a.F.}

In seiner Entscheidung geht das OLG München zunächst auf $\$ 9 \mathrm{HWG}$ a.F. (entspricht $\$ 9 \mathrm{~S} .1 \mathrm{HWG}$ n. F.) ein und stellt fest, dass die streitgegenständliche Werbung im Zeitpunkt ihrer Vornahme auf der Grundlage des $\$ 9 \mathrm{HWG}$ in der bis zum 19.12.1019 geltenden Fassung unzulässig war. Dabei vertritt das OLG München die Rechtsauffassung, dass $\$ 9 \mathrm{HWG}$ a. F. nicht dahingehend einschränkend auszulegen ist, dass das Werbeverbot akzessorisch die Unzulässigkeit der beworbenen Behandlung voraussetzt.

Folgt man dieser Rechtsauffassung und berücksichtigt man, dass seit der Liberalisierung der berufsrechtlichen Fernbehandlungsregelung in den Kammerberufsordnungen - infolge des Beschlusses des 121. Deutschen Ärztetages zur Änderung des $\$ 7$ Abs. 4 MBO-Ä im Mai 2018 - auch eine ausschließliche Fernbehandlung unter bestimmten, im Einzelfall geltenden Voraussetzungen erlaubt ist, führt dies zu dem (inkonsistenten) Ergebnis, dass es Ärzten - zumindest nach alter Rechtslage - zwar einerseits erlaubt war, Fern-

Syndikusrechtsanwalt Dr. iur. Julian Braun,

Fachanwalt für Medizinrecht,

Heartbeat Labs GmbH,

Rosa-Luxemburg-Straße 2, 10178 Berlin, Deutschland 
behandlung zu erbringen, ihnen andererseits aber verboten war, ihre Fernbehandlungsleistungen zu bewerben ${ }^{1}$.

Um diesen Widerspruch zu lösen, hätte das OLG München auch die genau gegenteilige Rechtsauffassung vertreten und entscheiden können, dass (schon) nach \$9 HWG a. F. (jetzt $\$ 9$ S. $1 \mathrm{HWG}$ ) die Werbung für berufsrechtlich zulässige Formen der Fernbehandlung erlaubt und nur die Bewerbung von unzulässigen Formen der Fernbehandlung verboten ist ${ }^{2}$. Dadurch wären das ärztliche Berufsrecht und das Werberecht hinsichtlich der Erbringung von Fernbehandlungsleistungen angeglichen und ein - wünschenswerter - Gleichlauf erzielt worden.

\section{Zum Verstoß gegen $\$ 9$ HWG n. F., insbesondere zur Ausnahmevorschrift des $₫ 9$ S. 2 HWG n.F.}

Im Rahmen der Prüfung des $\$ 9$ HWG n. F. stellt das OLG München zunächst fest, dass der Gesetzgeber das grundsätzlich geltende Verbot einer Werbung für Fernbehandlungen in $\$ 9$ S. $1 \mathrm{HWG}$ n. F. beibehalten hat und die Grundnorm des $\$ 9 \mathrm{HWG}$ a. F. somit weiterhin fortgilt. Betrachtet man deshalb allein die Regelung des $\$ 9$ S. 1 HWG n. F., so hat die oben dargestellte Inkonsistenz zwischen berufsrechtlich erlaubter ausschließlicher Fernbehandlung und dem geltenden Fernbehandlungswerbeverbot weiterhin Bestand.

Wichtig sind deshalb, die darauffolgenden Ausführungen des OLG München zur Neuregelung des $\$ 9$ S. 2 HWG und zur Prüfung, ob die streitgegenständliche Werbung ausnahmsweise zulässig ist.

Die Ausnahmevorschrift des $\$ 9$ S. 2 HWG n.F.$^{3}$. wurde im Rahmen des Digitale-Versorgung-Gesetzes (DVG) eingeführt. Gesetzgeberisches Ziel dieser Regelung ist es, die durch Beschluss des 121. Deutschen Ärztetages erfolgte Anpassung des ärztlichen Berufsrechts bzgl. der Erbringung von ausschließlicher Fernbehandlung im Hinblick auf die Reichweite des Werbeverbotes nachzuvollziehen und insoweit anzugleichen ${ }^{4}$. Allerdings hat der Gesetzgeber in \9 S. 2 HWG keine direkte bzw. akzessorische Kopplung zwischen Berufsrecht und Werberecht vorgenommen, sondern die Voraussetzungen, unter denen Werbung für Fernbehandlung gestattet ist, selbst geregelt ${ }^{5}$. Danach ist eine Werbung für Fernbehandlung erlaubt, ,wenn nach allgemein anerkannten fachlichen Standards ein persönlicher ärztlicher Kontakt mit dem zu behandelnden Menschen nicht erforderlich ist".

Dabei geht das OLG München - im Einklang mit der Gesetzesbegründung - davon aus, dass bei der Anwendung des $₫ 9$ S. 2 HWG eine abstrakt-generalisierende Bewertung erforderlich ist $^{6}$ und nur solche Fernbehandlungen bei Menschen beworben werden dürfen, bei denen die Einhaltung anerkannter fachlicher Standards gesichert ist, wenn also nach dem anerkannten medizinischen Stand der Erkenntnisse eine ordnungsgemäße Behandlung und Beratung unter Einsatz von Kommunikationsmedien grundsätzlich möglich ist ${ }^{7}$.

Bei der Auslegung des $\$ 9$ S. 2 HWG n.F. nimmt das OLG München eine eher konservative Perspektive ein und zeigt, dass es eine (ausschließliche) Fernbehandlung per se schon kritisch sieht. Besonders deutlich wird dies in der Aussage des OLG München, dass ,grundsätzlich jeder Krankheitsverdacht nach allgemeinen fachlichen Standards eine Basisuntersuchung erfordert, in der Regel unmittelbar durch Funktionsprüfungen (etwa von Atmung, Kreislauf, Blutdruck) und Besichtigungen, Abtasten, Abklopfen und Abhören des Körpers sowie ggf. der Erhebung weiterer Laborwerte“. Wäre diese Ansicht richtig, wäre jeder ausschließlichen Fernbehandlung der rechtliche Boden entzogen, weil es dann berufsrechtlich i.S.d. $\$ 7$ Abs. 4 MBO- $\ddot{A}$ weder ,,ärztlich vertretbar“, noch die ,,erforderliche ärztliche Sorgfalt" gewahrt wäre, wenn ein Arzt ohne (vorherigen) unmittelbaren physischen Patientenkontakt Fernbehandlungsleistungen erbringen würde. Schon dies spricht gegen die kritische Auffassung des OLG München. Richtigerweise muss es Ärzten möglich sein, einen Fernkontakt zu Patienten herzustellen, um im Rahmen dieses Kontakts den jeweiligen Einzelfall zu beurteilen und zu entscheiden, ob eine reine Fernbehandlung fachlich vertretbar oder stattdessen ein unmittelbar physischer Kontakt erforderlich ist.

Auf der Basis seiner oben geschilderten Annahme kommt das OLG München zum Ergebnis, dass die Bewerbung von Primärversorgungsmodellen, bei denen z.B. per App eine digitale ärztliche Fernbehandlung ,,von der Diagnose über die Therapieempfehlung bis hin zur Krankschreibung" für nicht näher konkretisierte Behandlungsfälle und -situationen angeboten wird, vom Ausnahmetatbestand des $\$ 9$ S. 2 HWG n.F. nicht gedeckt sei. Als Begründung führt dazu das OLG München insbesondere an, dass das grundsätzliche Werbeverbot für Fernbehandlungen nach \& 9 S. 1 HWG n. F. praktisch leerlaufen würde, wenn man die hier streitgegenständliche Werbung unter die Ausnahmeregelung des $₫ 9$ S. 2 HWG n. F. subsumieren wollte.

Der Entscheidung des OLG München ist weder im Ergebnis noch in seiner Begründung zuzustimmen.

Einerseits intendiert der Gesetzgeber mit $\$ 9$ S. 2 HWG n. F., dass Ärzte (auch) für Online-Sprechstunden werben, d.h. darüber auf ihren Internetseiten informieren dürfen ${ }^{8}$. Da es sich auch bei Online-Sprechstunden um Primärversorgungsmodelle handelt, ist davon auszugehen, dass die Bewerbung einer solchen Online-Sprechstunde an sich d.h. auch ohne konkrete Benennung näher konkretisierter Behandlungsfälle und -situationen - auch bei abstrakt-generalisierender Bewertung zulässig sein muss ${ }^{9}$.

Andererseits ist es gerade andersherum, als das OLG München meint. Würde man die Werbung für Fernbehandlungs-Primärversorgungsmodelle - zu denen wie gesagt auch Online- bzw. Videosprechstunden gehören - stets als verboten ansehen, bliebe im Rahmen des $\$ 9$ S. 2 HWG n. F. kein Raum mehr, um ausschließliche ärztliche Fernbehandlung in zulässiger Weise zu bewerben.

Bei der Auslegung des $\$ 9$ S. 2 HWG n. F. ist richtigerweise kein allzu strenger Maßstab anzulegen, weil ansonsten die Gefahr besteht, dass dem gesetzgeberischen Willen, mit der Einfügung des $\$ 9$ S. 2 HWG einer Weiterentwicklung telemedizinischer Möglichkeiten Rechnung $\mathrm{zu}$ tragen $^{10}$, nicht entsprochen werden $\mathrm{kann}^{11}$. Die in $\$ 9 \mathrm{~S}$. $2 \mathrm{HWG}$ festgelegten Anforderungen an die Zulässigkeit der Werbung für Fernbehandlung sind niedrig zu halten, um nicht mit einem zu strengen Werberecht die Liberalisierung des

1) So schon Braun, MedR 2018, 563, 566; vgl. auch Kuhn/Heinz, GesR 2018, 691, 694; Eichelberger, in: Ahrens/Büscher/Goldmann/ McGuire (Hrsg.), FS f. Henning Harte-Bavendamm, 2020, S. 289, 296.

2) So Braun, MedR 2018, 563, 566; ebenso Spickhoff, MedR 2018 , 535, 542; Eichelberger, in: Ahrens/Büscher/Goldmann/McGuire (Hrsg.), FS f. Henning Harte-Bavendamm, 2020, S. 289, 296 mit eingehender verfassungsrechtlicher Begründung.

3) Zum Charakter des $\$ 9$ S. 2 HWG als Ausnahmeregelung vgl. auch Tillmanns, A\&R 2020, 11, 13.

4) BT-Dr. 19/13438, S. $77 \mathrm{ff}$

5) Darauf weist Eichelberger, in: Ahrens/Büscher/Goldmann/McGuire (Hrsg.), FS f. Henning Harte-Bavendamm, 2020, S. 289, $298 \mathrm{zu}-$ treffend hin.

6) Vgl. dazu Tillmanns, A\&R 2020, 11, 14; Eichelberger, in: Ahrens/ Büscher/Goldmann/McGuire (Hrsg.), FS f. Henning Harte-Bavendamm, 2020, S. 289, 298

7) BT-Dr. 19/13438, S. 78.

8) Vgl. dazu Pressemitteilung des Bundesministeriums für Gesundheit v. 10.7.2019, abzurufen unter https://www.bundesgesundheitsministerium.de/presse/pressemitteilungen/2019/3-quartal/ dvg-kabinett.html (zuletzt abgerufen am 3.9.2020).

9) So auch zutreffend Tillmanns, A\&R 2020, 11, 14.

10) BT-Dr. 19/13438, S. 78.

11) So auch Tillmanns, A\&R 2020, 11, 14 
ärztlichen Berufsrechts zu konterkarieren ${ }^{12}$. Deshalb sollte es auch bei abstrakt-genereller Betrachtungsweise genügen, dass für die beworbene Fernbehandlung ernsthaft praktisch relevante Anwendungsfälle bestehen oder bestehen können ${ }^{13}$. Dies ist bei Primärversorgungsmodellen auch dann der Fall, wenn in der Werbung keine konkreten Behandlungsfälle und -situationen genannt werden. Insbesondere bei bloßen Erkältungserscheinungen oder im Bereich der Dermatologie, die sich v.a. auf die Inaugenscheinnahme von äußeren Unregelmäßigkeiten der Haut stützt, bestehen praktisch relevante Anwendungsfälle für Fernbehandlungen ohne (vorherigen) physischen Arzt-Patienten-Kontakt, die gem. $\int 7$ Abs. $4 \mathrm{MBO}-\ddot{\mathrm{A}}$ in rechtlich zulässiger Weise durchgeführt werden können.

Soweit das OLG München andeutet, dass eine Fernbehandlungswerbung für Primärversorgungsmodelle mit näher konkretisierten Behandlungsfällen und -situationen werberechtlich liberaler zu beurteilen seien, geht dies ebenfalls fehl. Eine Beschränkung des Fernbehandlungsangebots auf bestimmte Indikationen oder Untersuchungen würde voraussetzen, dass seitens der Patienten (Werbeadressaten) ein ausreichend medizinisches Verständnis besteht, nach dem er selbst beurteilen kann, ob seine aktuellen Gesundheitsbeschwerden in diese Anwendungsbereiche fallen oder nicht. Dies pauschal anzunehmen ist aber unsicher. Stattdessen ist es gerade Aufgabe der Ärzte, den gesundheitlichen Einzelfall medizinisch zu beurteilen, so dass sich eine Abwälzung dieser Beurteilung auf die Patienten verbietet Da eben diese ärztliche Beurteilung berufsrechtlich auch geboten ist, kann mit Blick auf den Telos des $\$ 9$ S. 1 HWG n.F. keine Gesundheitsgefahr erkannt werden, die durch Werbung für Fernbehandlungen befördert würde.

Es ist auch im Übrigen nicht richtig, dass das grundsätzliche Werbeverbot für Fernbehandlungen nach \$9 S. $1 \mathrm{HWG}$ n. F. leerlaufen würde, wenn Werbung für ausschließliche ärztliche Fernbehandlungsangebote unter die Ausnahmeregelung des $₫ 9$ S. 2 HWG n. F. subsumiert würde. Bei der Schaffung des Ausnahmetatbestands des $₫ 9$ S. 2 HWG kam es dem Gesetzgeber gerade darauf an, die Werbung für ärztliche Fernbehandlung weitgehend zuzulassen und nur noch die Bewerbung von Fernbehandlungen, die durch Personen angeboten werden, bei denen weder die Fernbehandlung noch das Bewerben der Fernbehandlung durch eine rechtlich verbindliche Berufsordnung geregelt werden, dem Verbotstatbestand des $\$ 9$ S. 1 HWG zu unterwerfen ${ }^{14}$. Da Heilpraktiker keinem rechtlich verbindlichen Berufsrecht unterliegen ${ }^{15}$, bleibt z.B. die Werbung für Fernbehandlung durch Heilpraktiker vom Werbeverbot des $\$ 9$ S. 1 HWG erfasst. Das gleiche gilt für die Werbung für Fernbehandlung durch Tierärzte, weil die Ausnahmevorschrift des $\$ 9 \mathrm{~S}$. $2 \mathrm{HWG}$ auf die Werbung für Fernbehandlung am Menschen ${ }^{16}$ beschränkt ist.

\section{Zum Verstoß gegen $\$ 9$ HWG n.F. bei Werbung für Krankschreibungen im Wege von Online-Videokonsultationen}

Schließlich nimmt das OLG München noch besonderen Bezug auf die streitgegenständliche Werbung Krankschreibungen im Wege der Online-Videokonsultation. Dazu führt das OLG München unter Verweis auf $\$ 25 \mathrm{~S} .1 \mathrm{MBO}$ $\ddot{A}$ aus, dass nach allgemeinen fachlichen Standards ein persönlicher ärztlicher Kontakt mit dem zu behandelnden Menschen - gerade bei Patienten, die dem Arzt persönlich unbekannt sind -i. S.d. \$9 S. 2 HWG grundsätzlich erforderlich ist, so dass die Werbung für generelle Krankschreibungen per Videokonsultation nicht auf $\int 9$ S. 2 HWG gestützt werden könne.

Dies überzeugt nicht. Es ist - anders als das OLG München ausführt - insbesondere nicht richtig, dass nach allgemeinen fachlichen Standards - vor Ausstellung einer Arbeitsunfähigkeitsbescheinigung - ein persönlicher phy- sischer Arzt-Patienten-Kontakt grundsätzlich erforderlich ist. Dies gilt auch dann nicht, wenn der jeweilige Patient dem Arzt unbekannt ist.

Im juristischen Schrifttum wurde schon ausführlich analysiert und dargestellt, dass im Rahmen der \$\$ 7 Abs. 4, 25 S. $1 \mathrm{MBO}-\ddot{A}$ Arbeitsunfähigkeitsbescheinigungen auch im Wege von ausschließlicher Fernbehandlung ausgestellt werden können und dürfen ${ }^{17}$. Dabei wurde zuletzt auch im Besonderen darauf hingewiesen, dass infolge der Änderungsvereinbarungen zu $₫ 31 \mathrm{BMV}-\ddot{A}-$ COVID 19 v. 9.3.2020 und 23.3.2020 und der weiteren Zusatzvereinbarung v. 11.3.2020 die apodiktische Behauptung, die Ausstellung von Arbeitsunfähigkeitsbescheinigungen verstoße in jedem Fall gegen die ärztliche Sorgfalt, vom Tisch sein dürfte ${ }^{18}$.

Auch die Bundesärztekammer geht davon aus, dass die Feststellung von Arbeitsunfähigkeit und die Ausstellung einer entsprechenden ärztlichen Bescheinigung im Rahmen ausschließlicher Fernbehandlung berufsrechtlich vorstellbar ist, wenn der ausstellende Arzt im Einzelfall auf Grundlage der (ausschließlich) über Kommunikationsmedien übermittelten Informationen zur Überzeugung gelangt, dass die Ausstellung der ärztlichen Bescheinigung vertretbar ist und die in der Bescheinigung enthaltenen Feststellungen zutreffen ${ }^{19}$.

12) So ausdrücklich Eichelberger, in: Ahrens/Büscher/Goldmann/McGuire (Hrsg.), FS f. Henning Harte-Bavendamm, 2020, S. 289, 299

13) Eichelberger; in: Ahrens/Büscher/Goldmann/McGuire (Hrsg.), FS f. Henning Harte-Bavendamm, 2020, S. 289, 299.

14) BT-Dr. 19/13438, S. 77.

15) Dazu Eichelberger, in: Ahrens/Büscher/Goldmann/McGuire (Hrsg.), FS f. Henning Harte-Bavendamm, 2020, S. 289, 300 m. w. N.

16) Vgl. Wortlaut des $\$ 9$ S. 2 HWG: ,[ ] dem zu behandelnden Menschen $[\ldots]^{*}$

17) Vgl. dazu insbesondere Braun, GesR 2018, 409; Hahn, MedR 2020, 370; Hahn, ZMGR 2018, 279.

18) So ausdrücklich und zutreffend Hahn, MedR 2020, 370, 374.

19) $\mathrm{BÄK}$, Hinweise und Erläuterungen zu $\$ 7$ Abs. $4 \mathrm{MBO}-\ddot{\mathrm{A}}-\mathrm{Be}-$ handlung im persönlichen Kontakt und Fernbehandlung, Stand: 22.3.2019, abzurufen unter: https://www.bundesaerztekammer.de/ fileadmin/user_upload/downloads/pdf-Ordner/Recht/Hinweise ErlaeuterungenFernbehandlung.pdf (zuletzt abgerufen 4.9.2020); BÄK, Fragenkatalog zur Fernbehandlung, Stand: 5. 4.2019, abzurufen unter: https://www.bundesaerztekammer.de/recht/publikationen/fragenkatalog/ (zuletzt abgerufen 4.9.2020).

\section{Zur Frage, ob durchgangsärztliche Fehler im hoheitlichen Bereich nach Übernahme der Behandlung fortwirken (I.)}

\section{GG Art. 34; BGB §630a; SGB VII §34}

1. Bei der vom Durchgangsarzt zu treffenden Entscheidung, ob die allgemeine oder die besondere Heilbehandlung erforderlich ist, handelt es sich um eine der Berufsgenossenschaft obliegende öffentlich-rechtliche Aufgabe; für Fehler des Durchgangsarztes in diesem Bereich haftet dieser nicht persönlich.

2. Bleibt eine Beschwerdeverbesserung aus, kann ein Behandler verpflichtet sein, ein (initial falsch) vorbewertetes Röntgenbild erneut in Augenschein zu nehmen und $\mathrm{zu}$ bewerten, weil ein gedacht in der Zwischenzeit die Behandlung übernehmender, dritter Arzt hierzu ebenso verpflichtet wäre.

Eingesandt und bearbeitet von Rechtsanwalt Dr. iur. Thorsten Süß, Fachanwalt für Medizinrecht,

BLD Bach Langheid Dallmayr Rechtsanwälte

Partnerschaftsgesellschaft $\mathrm{mbH}$,

Theodor-Heuss-Ring 13-15, 50668 Köln, Deutschland 